\title{
INVASIVE PULMONARY ASPERGILLOSIS
}

\section{IN COPD EXACERBATION}

BULPA P. ${ }^{1}$, DUPLAQUET F. ${ }^{2}$, DIMOPOULOS $\mathrm{G}^{3}$, VOGELAERS $\mathrm{D}^{4}$, BLOT S. ${ }^{5}$

Address for correspondence:

${ }^{1}$ Bulpa Pierre, MD

Intensive Care Unit

Mont-Godinne University Hospital, CHU UCL Namur

Avenue Thérasse, 1, 5530 YVOIR, Belgium

pierre.bulpa@uclouvain.be

Phone : $32(0) 814238$ 63; Fax +32(0)81423862

Co-authors

${ }^{2}$ Duplaquet Fabrice, MD

Pneumology

Mont-Godinne University Hospital, CHU UCL Namur

Avenue Thérasse, 1, 5530 YVOIR, Belgium

fabrice.duplaquet@uclouvain.be

Phone: + 32 (0)81 4233 51; Fax: + 32 (0)81 423352

${ }^{3}$ Dimopoulos George, MD, PhD

Department of Critical Care Medicine

Attikon University Hospital, 1 Rimini Str., 124 62, Haidari, Greece

gdimop@med.uoa.gr

Phone: ++30 (210) 583-2182; Fax: +30 (210) 583-2182 
${ }^{4}$ Vogelaers Dirk, MD, PhD

Department of Internal Medicine and Pediatrics

Ghent University, Corneel Heymanslaan 10- 9000 Ghent, Belgium

dirk.vogelaers@uzgent.be

Phone: +32 (0)9 33254 78;

${ }^{5}$ Blot Stijn, MNSc, PhD

Department of Internal Medicine and Pediatrics

Ghent University, Corneel Heymanslaan 10- 9000 Ghent, Belgium

Stijn.blot@ugent.be

Phone: + $32(0) 93226216$

Keywords: Aspergillus, invasive pulmonary aspergillosis, COPD, exacerbation 


\section{ABSTRACT}

Nowadays, reports in the literature support that patients with severe COPD are at higher risk to develop invasive pulmonary aspergillosis (IPA). However, the interpretation of Aspergillus-positive cultures from the airways in critically ill COPD is still a challenge. Indeed, as the patient could be merely colonized, tissue samples are required to ascertain IPA diagnosis but they are rarely obtained before death. Consequently, diagnosis is often only suspected on the basis of a combination of three elements: clinical characteristics, radiological images (mostly thoracic ct-scan), microbiological, and occasionally serological, results. To facilitate the analysis of these data, several algorithms have been developed, and the best effectiveness has been demonstrated by the Clinical algorithm. This is of importance as IPA prognosis in these patients remains presently very poor and using such algorithm could promote prompter diagnosis, early initiation of treatment and subsequently improved outcome.

While the most classical presentation of IPA in critically ill COPD patients features a combination of an obstructive respiratory failure, an antibiotic resistant pneumonia, recent or chronic corticosteroids therapy, and positive Aspergillus cultures from the lower respiratory tract, the present manuscript will also address less typical presentations and discuss the most appriopriate treatments which could alter prognosis. 


\section{INTRODUCTION}

Chronic obstructive pulmonary disease (COPD) patients frequently experience acute exacerbations (AECOPD) which may require hospitalization. These AECOPD most often link to an infectious origin, mainly viral or bacterial. However, reports have focused on Aspergillus infections as a possible cause for acute respiratory deterioration, essentially in COPD patients under steroid therapy [1]. Due to progressive respiratory failure, these patients often require intensive care unit (ICU) admission for ventilatory support.

Contrasting with oncohematological patients [2], data remain limited in the literature, but recent epidemiologic and autopsy data have been published. As in haematological and solidorgan transplant patients [3], mortality rate remains high [1, AspICU project, data on file [4]]. Thus, an early diagnosis and initiation of treatment seem key to enhance prognosis [5]. But, as the presence of Aspergillus spp. in the airways does not equate to infection [1], the diagnosis of invasive pulmonary aspergillosis (IPA) remains a challenge and consequently IPA diagnosis habitually relies on a cluster of clinical, laboratory and radiological findings. Therefore, this review will discuss the epidemiology, clinical signs, laboratory findings and diagnostic procedures to facilitate rapid recognition of IPA in COPD patients in order to initiate antifungals as early as possible. 


\section{IPA DEFINITIONS:}

Clinical diagnosis of IPA remains a challenge, as the most frequently used diagnostic criteria (defined by the European Organization for Research and Treatment of Cancer and Treatment of Cancer/Mycosis Study Group [EORTC/MSG]) were developed for research purposes and not for daily clinical practice. Moreover these definitons focus on the classic 'at risk' population with haematological and oncological malignancies and are, as such, not appropriated for COPD or critically ill patients [6]. To address this issue, the EORTC/MSG algorithm was recently adapted twice resulting in the elaboration of the algorithm for COPD patients (COPD algorithm) [1] and the Clinical algorithm for ICU patients [7, 8]. The latter was subsequently externally validated in a large population of ICU patients [4], and the three were compared in a large cohort of ICU patients with COPD. In that cohort, the Clinical algorithm demonstrated higher accuracy to detect IPA than the COPD one. In turn, both algorithms had greater diagnostic value than the EORTC/MSG criteria [9]. Table1 


\section{EPIDEMIOLOGY}

Despite increasing cases reported in the literature, the incidence of IPA in COPD patients remains poorly documented. This is mainly due to the lack of a consistent case definition and the absence of specific surveillance efforts. In order to facilitate the diagnosis, several authors have developed diagnostic criteria $[1,4,6]$, but their use is not yet generalized. In addition, as the growth of Aspergillus spp. can be interpreted as mere colonization in COPD patients, IPA diagnosis could be delayed [1]. A large review reported that about $1 \%$ of patients with invasive aspergillosis (IA) suffered from COPD [1]. However, in a large study of IPA in ICU patients, COPD was the underlying condition in $34 \%$ of patients suffering from IPA [10]. In a more recent autopsy study, IPA was diagnosed in $2.8 \%$ of patients while $44 \%$ suffered from

COPD [11]. Finally, Guinea et al. reported a growth of IPA cases among these patients (from $0.7 \%$ in 2000 to $1.3 \%$ in 2007 ) [12].

Correlation between the daily dose of corticosteroids and the probability to develop IA was investigated by several authors. Pooling the data from the literature, more than $20 \mathrm{mg}$ prednisone per day or a cumulative dose of more than $700 \mathrm{mg}$ was associated with an increased risk of infectious complications [13]. However, for COPD patients, precise dosages or durations of corticotherapy as a risk factor for IPA cannot be deduced from the literature, but risk for IPAhas been associated with high doses and/or prolonged administration of steroids [14-17]. Additionally, some reports have suggested that high-doses of inhaled corticosteroids may also promote IPA [18-20]. Therefore, in severe COPD, the risk of side effects of inhaled steroids (particularly infections related to immune system perturbances) must be weighed against the expected and well proven respiratory benefits. Furthermore, COPD patients can as well develop IPA without prior corticosteroids exposure [21]. 
In addition, there is now a growing body of evidence to support that infections, in particular viral infection such as influenza [22, 23] or cytomegalovirus (CMV) may promote IPA [24]. 


\section{PATHOPHYSIOLOGY}

Aspergillus spp. is a worldwide airborne pathogen with spores small enough ( 2 to $3 \mu \mathrm{m}$ ) to reach lung parenchyma via the airways. In COPD patients, the defence mechanisms of the airways, which normally clear out the majority of Aspergillus conidia, are impaired by tobacco smoke (through alteration of the ciliary activity of the bronchial epithelium), and by repeated epithelial damages due to iterative episodes of infection. These lesions enable the binding of conidia to the epithelial layer [25]. Thus, in contrast to haematological patients where Aspergillus dissemination is a bloodborne process, the bronchial mucosa is first invaded, then the contiguous lung parenchyma, and finally the vasculature inducing secondary pulmonary infarction.

The immune system, especially alveolar macrophages (AM) and neutrophils, fulfills a key role in the destruction of the A. fumigatus conidia principally by AM and of the hyphae and germinating spores by neutrophils [26-30].

Mechanisms and sequence of the conidia destruction by AM have been extensively investigated [30-33]. By inhibiting AM and neutrophils antifungal activities, steroids facilitate the onset of IPA, and, by themselves, boost the growth of some Aspergillus spp. (especially $A$. fumigatus) [33-36]. Additionally, corticosteroids modify the TH1-TH2 cytokines response to infection and thus reduce the defences against Aspergillus [37].

In an experimental model with corticosteroid-exposed animals, histological examinations of IPA lesions in the lung parenchyma showed large foci of pneumonia, exudative bronchiolitis with bronchial and alveolar destructions, and haemorrhagic necrosis with neutrophil infiltration [29, 38], with only few Aspergillus detected and, when present, typically in their nongerminated form. Thus, the corticosteroid-treated animals, unlike neutropenic ones, appear to die from a disproportionate host reaction instead from the fungal invasion itself. These 
observations could enlighten, at least partially, why pneumonia is the main lesion identified in histological examinations, and therefore that, in corticosteroid-treated COPD patients, chest X-rays more often demonstrate nonspecific consolidations than multiple nodules [1]. In addition, in these patients, haematogenous metastasis seems unusual as both autopsy studies in COPD patients and findings from IPA models in animals under corticosteroids, finally, rarely reported disseminated aspergillosis $[1,29,38]$.

Furthermore, in the setting of sepsis and septic shock, which remains one of the most frequent causes for ICU referral, the immune response is biphasic and could explain why some COPD patients without apparent immunodepression may acquire IPA. Indeed, sepsis is divided into two phases: an initial hyperinflammatory response followed by an anti-inflammatory phase. When the latter reaction is disproportionate, it leads to the known compensatory antiinflammatory response syndrome (CARS) characterized by immunoparalysis. As a consequence, the patient is temporarily immunocompromised and thus at increased risk for opportunistic infections such as IPA [39]. 


\section{CLINICAL FEATURES}

The most frequently reported clinical sign in COPD patients with IPA is a nonspecific antibiotic-resistant pneumonia associated with exacerbated dyspnoea [1]. The analysis from a large database on ICU patients with Aspergillus-positive cultures confirmed these data (AspICU project, data on file [4]). Indeed, depending on the algorithm used, 52 to $68 \%$ of patients with IPA required invasive ventilation compared to 2 to $24 \%$ of the patients considered to be merely colonized by the algorithm.

Patients' characteristics and clinical features reported in the largest review on COPD patients suffering from IPA are summarized in table 2. At hospital admission, 77\% of patients were under corticosteroids (including inhaled forms); this rate amounts to $96 \%$ during hospitalization. The evolution of symptoms is characterized by an improvement of the respiratory signs followed by an iterative recrudescence of the dyspnea $[15,17]$, featured by a predominant bronchospastic component $[1,12,40]$. In contrary to haematological patients [41], fever, chest pain and haemoptysis were less often encountered in the COPD population [12]. These data were subsequently supported by a large database [4] of ICU COPD patients (AspICU project, data on file).

It takes a median of 8.5 days between onset of IPA related symptoms and diagnosis. This long delay may have contributed to the high rate of need for invasive ventilation ( $77 \%$ of patients) $[1]$.

Although aspecific, an elevated white blood cell count higher than 12,000/ml was described in $75 \%$ of patients as well as thrombocytopenia $\left(<100,000\right.$ platelets $\left./ \mathrm{mm}^{3}\right)$ in the late course [15]. On the other hand, frequently dosed markers, such as C-reactive protein, procalcitonin, fibrinogen and lactate dehydrogenase, are not systematically raised and therefore offer no discerimination. 
Consequently, the optimal way to diagnose IPA in COPD remains to be determined especially when the patients are intubated and mechanically ventilated. Indeed, open lung biopsy might be contraindicated due to coagulation disorders and/or severe respiratory failure [42, 43], precluding the possibility to prove IPA. To date, three algorithms have been published to diagnose IPA: one is specific for COPD [1] whereas the two others are more generic (Clinical [8] and EORTC/MSG [6] algorithms). But the latter has been validated only in immunocompromised patients; however ICU COPD patients only fulfill the classic host factors criteria with the EORTC/MSG algorithm when exposed to corticosteroid therapy for a prolonged period of time, defined as $0.3 \mathrm{mg} / \mathrm{kg} /$ day of prednisone equivalent for $>3$ weeks [6, 44]. Blot and colleagues have reported that the Clinical algorithm detects a greater proportion of the disease burden in ICU patients compared with the EORTC/MSG criteria mainly because host factor was often lacking in ICU patients [4]. A recent study on a large ICU COPD population confirmed that more IPA cases were detected with the Clinical algorithm as compared to the EORTC/MSG criteria $(\mathrm{p}=0.013)$ [9]. As the definitions for proven IPA are equal, the observed differences lie within the chosen diagnostic approach. Indeed, for both COPD and EORTC/MSG algorithms, a number of patients could not be classified: for the former because only GOLD III or IV could be considered as probable IPA, and for the latter as COPD is not recognized, in itself, as a relevant host risk factor. In addition, concordance between algorithms was assessed in 23 patients with histological data. A correct diagnosis (True Positive + True Negative) was more often obtained with the Clinical followed by the COPD and finally the EORTC/MSG algorithm (19, 12, 3) [9]. Finally, analysing the 8 patients in whom IPA was discovered only after death, the Clinical, COPD and EORTC/MSG algorithms classified them as putative/probable IPA in 7, 4 and 0 of the cases, respectively. Another insight from the AspICU study is that GOLD II classified COPD patients under corticosteroids could be subject to developing IPA $[9,40]$. 
Therefore, in severe steroid-dependent COPD patients, the presence of a dyspnoea exacerbation and poor clinical status, despite broad-spectrum antibiotics and high doses of steroids, is highly suggestive of IPA, especially when a recent pulmonary infiltrate appears on Chest X-ray and/or when Aspergillus species is retrieved in the sputum.

Hence, among COPD patients admitted to ICU, the Clinical algorithm seems to be more useful to diagnose IPA. The COPD algorithm is less appropriate due to the number of nonconclusive diagnoses in particular for GOLD II COPD patients. IPA should be considered in COPD patients, regardless of the GOLD stage, under chronic corticosteroid therapy with Aspergillus isolation, respiratory failure and abnormal chest X-ray, especially after broadspectrum antibiotic therapy. When IPA is probable and hence not yet confirmed, additional investigations must be undertaken to confirm the infection and treatment should be formally taken into account.

\section{DIAGNOSTIC PROCEDURES}

\section{$\underline{\text { SPUTUM }}$}

The exact incidence of airways colonization by Aspergillus spp. in the COPD population remains to be determined [45-48], but a recent report suggested that $78 \%$ of their patients were [12]. Nevertheless, as pointed out above, when faced with antibiotic resistant pneumonia, a positive culture for Aspergillus spp. must alert the physician.

Direct examination for Aspergillus spp., readily accessible as sputum production is generally increased in case of IPA, is positive in $48 \%$ in this context [49]. Using the fluorescence techniques, positivity may increase up to $88 \%$ [50]. Although, in COPD patients, the presence of Aspergillus does not ascertain IPA diagnosis, it must raise the suspicion of IPA particularly 
when a pulmonary infection proves unresponsive to antibiotics, but not exclusively, in case of corticotherapy. Repetitively positive cultures are even more indicative of infection [51-53], even if bacteria are cultured, which is often the case [1]. Nonetheless, the issue of diagnostic performance of sputum analyses remains debated in the literature [1]. The explanation could be that colonization may reflect a transitory passage of Aspergillus in the tracheo-bronchial tree, a prolonged but benign carriage, but also a necessary first step prior to invasive disease (as the incubation period before IPA is not determined) [54]. Yet, the physician should keep in mind that an IPA may be present even in the absence of a positive culture for Aspergillus $[1]$.

\section{$\underline{\text { SEROLOGY }}$}

To date, the galactomannan (GM) antigen test (ELISA test, Platelia $\left.{ }^{\circledR}\right)$ was the most often diagnostic test used in clinical practice and predominantly studied in hematological cancers. One of the main interests lies in the fact that diagnosis could be obtained before appearance of clinical signs $[55,56]$, if blood samples were taken at least twice weekly with an optical density index (ODI) set to 1 . However, other authors did not confirm these results [57-59]. A recent meta-analysis reviewed numerous studies evaluating the performance of the test $[60$, 61]. Some noted discrepancies are due to the number of tests performed per week, the definition of IPA used or analysed populations, running antifungals, as well as the ODI cutoff chosen. In the above-mentioned meta-analysis including 5660 immunocompromised patients, mostly hematological, counting 586 with proven or probable invasive aspergillosis, using an OD index cutoff of 0.5 , sensitivity was 82 percent (95\% CI 73-90 percent) and specificity 81 percent (95\% CI 72-90 percent) [61].

Few studies specifically assessed antigenemia in critically ill COPD patients suspected to have IPA. Despite several biases and with ODI set to 0.5 , sensitivity was reported between 11 
to $91 \%$ and specificity 66 to $100 \%[44,62-64]$. It has been suggested that GM test was more efficacious in hematological population as GM is not cleared by leucocytes unlike what is happening in non immunocompromised individuals.

Again, data from hematological patients suggest that a rise in antigenemia of 1.0 within the first week could denote a progression of the infection which could be even more relevant for diagnosis than one value upper the normal level [65]. In addition, even if the therapy is active, pulmonary lesions secondary to Aspergillus spp. may grow on CT scan up to two weeks after the treatment beginning [66], thus, an increase of antigenemia could suggest a treatment failure inciting a change in antifungal therapy.

Nonetheless, despite some encouraging results, and specifically in COPD population, no decrease in mortality has been reported. Additional factors could also complicate the interpretation of the Platelia ${ }^{\circledR}$ test: antifungals could reduce the sensitivity of test [61], some cross-reactive antigens from foods and other intestinal fungi or bacteria could falsely positivate the galactomannan analysis $[67,68]$ or the simultaneous intravenous administration of piperacillin-tazobactam or amoxicillin-clavulanate [69] or plasmalyte [70]. For these latters, new products cross-react less with the assay [71].

Next to serologic tests, PCR analyses and dosage of plasma concentration of the beta-Dglucan, a component of the fungal cell wall, are available. In a meta-analysis, Cruciani et al. reported a mean sensitivity of $80.5 \%(95 \%$ CI 73.0 to 86.3$)$ and a mean specificity of $78.5 \%$ (95\% CI 67.8 to 86.4 ) with one PCR dosage to diagnose IPA [72]. When two consecutive positive tests are used, the mean sensitivity was $58.0 \%$ (95\% CI 36.5 to 76.8$)$ and the mean specificity $96.2 \%$ (95\% CI 89.6 to 98.6 ). To interpret these results, caution is required: definitions of IPA vary between studies, molecular methods are not standardized, plasma products could inhibit PCR testing, and the influence of previous antifungal exposure is unknown but a priori less sensitive to treatment pressure. Moreover, as the patients reported in 
the meta-analysis primarily suffered from hematological diseases, results could not be generalized particularly to COPD individuals where the difference between Aspergillus colonization and IPA is crucial but not robust enough with PCR. The detection of the beta-Dglucan in the plasma does not seem to be superior to determination of blood GM [73].

\section{$\underline{R A D I O L O G Y}$}

Related IPA radiological findings in COPD patients are quite different compare to the ones observed in hematological patients. Where non specific consolidations [fig 1,2] is the rule in the formers $[1,9,12,40]$, more suggestive images could be also seen but are specially described in the latters such as wedge shaped consolidation [fig 3], nodules [fig 4], "halo sign" [fig 3] (better seen on thoracic CT-scans) and "air-crescent sign" [fig 5]; these two latter being nearly pathognomonic of IPA in neutropenic haematological patients. In contrast, in COPD individuals, a normal chest X-ray virtually excludes IPA probably because pneumonia was diagnosed tardily as suggested by the high number of respiratory failure and intubation rate in patients suffering from IPA [9]. Interestingly, on thoracic CT-scan, wedge-shaped infiltrates, although rare, was nearly pathognomonic for IPA but did not reach statistical significance. Albeit more common, well-shaped nodules were particularly described in COPD patients with putative IPA [AspICU data on file [4]]. Again, in hematological population,

Caillot et al. showed that prompt performed thoracic CT scan, as it detects pulmonary lesions earlier than chest X-rays, could improve survival [5].

To avoid misdiagnosis, the clinician must keep in mind that nodules can have infectious as well as noninfectious origin such as malignancy. 


\section{FIBEROPTIC BRONCHOSCOPY}

Not every patient could provide sputum for conclusive analysis. To alleviate this, fiberscopy could be performed to obtain endotracheal aspirate, BAL, bronchial washing or brushing specimen for culture. Endotracheal aspirate culture yields were 46 to $77 \%$ [1, 38, 60], with a lower sensitivity in patients on antifungals [74]. BAL was more often positive when prolonged pneumonia or extensive involvement of the pulmonary parenchyma is present. Interestingly, cytological scrutiny seems to be even more positive than cultures $[8,53]$. It remains that a positive microscopy for Aspergillus alone could not absolutely distinguish colonization from infection. Currently, the detection of galactomannan in BAL fluid is recommended to diagnose IPA [75] using a cut-off index of 0.5 , with a sensitivity and a specificity of $87 \%(95 \%$ CI $0.79-0.92)$ and $89 \%$ (95\% CI 0.85-0.92) respectively [62-64, 74, 76].

However, whereas serum ELISA could be executed without lung infiltrates, BAL could be only performed in case of abnormal chest imaging and could not be repeated every day. Therefore, the ELISA test on serum could be more useful. In addition, usefulness of PCR tests on BAL fluid is still waiting as: standardization is still lacking and due to their high sensitivity difference between infection and colonization could not always be determined [77]. Whatever, the Avni's meta-analysis reported a sensitivity of $90.2 \%$ (95\% CI 77.2 to 96.1 ) and a specificity of $96.4 \%(95 \%$ CI 93.3 to 98.1$)$ [74].

Beyond the realization of culture, antigenic tests and PCR, performance of flexible bronchoscopy could detect some mucosal lesions enabling biopsies [fig 6], allowing diagnosis in $47 \%$ of the case [1]. Additionally, transbronchial lung biopsies (TBLB) can be executed but their efficacy only is around $25 \%$ [1]. Regrettably, flexible bronchoscopy could not always be performed in COPD patients related to their reduced lung status or function. 


\section{LUNG BIOPSY}

As stated previously, IPA can only be ascertained by histology. The ways to obtain lung biopsies are through fiberscopy (bronchial or transbronchial specimen) or more classicaly by open lung biopsy (OLB). Moreover, OLB may be of therapeutic interest by itself as pulmonary resection may be curative [5] (see surgical treatment). However, as lung function could be severely altered in COPD patients, surgery may be impracticable. Although lung biopsy could be achieved by percutaneous needle, with a success around 50\% (thus negativity does not exclude IPA), only peripheral lesions are concerned but in COPD individuals the likelihood of pneumothorax is increased [1].

While definitive confirmation of infection requires biopsy, immediate initiation of antifungals should be considered when IPA is suspected, lung biopsies being reserved for unconvincing cases. 


\section{THERAPY}

The data on treatment efficacy came mainly from hematological population thus are extended to COPD patients in the absence of dedicated studies. On this basis, when IPA is suspected, antifungals will be started without delay if compatible lesion(s) are detected on thoracic CT scan [41]. At the same time, if exposed to steroids, they will be lessened, or even stopped, to the smallest possible dose as steroids have been described as a risk factor to develop IPA.

\section{CURATIVE TREATMENTS}

Nowadays, amphotericin B deoxycholate (AmB-d) and itraconazole are no longer recommended as treatment against IPA. The former due to its related toxicity, mainly as at least $30 \%$ of patients are exposed to renal failure, the second because of associated drug interactions and poor bio-availability. Furthermore, studies have demonstrated a better efficacy of more recent antifungal compounds [78].

Today, voriconazole (VRC) is the antifungal of choice because it was the most studied and was the most effective $[78,79]$. The larger study, comparing VRC (two intravenous doses of $6 \mathrm{mg} / \mathrm{kg}$ on day 1 then $4 \mathrm{mg} / \mathrm{kg} / 12 \mathrm{~h}$ for at least 7 days, followed by $200 \mathrm{mg} / 12 \mathrm{~h}$ orally) against AmB-d (intravenously 1 to $1.5 \mathrm{mg} / \mathrm{kg} / \mathrm{d}$ ) in a randomized, unblinded trial that enrolled 277 patients, reported a survival rate at 12 weeks of $70.8 \%$ vs $57.9 \%(p=0.02)$ with a better tolerance of VRC [78]. Another advantage is that VRC is available in intravenous and oral forms. However, its usage may be limited due to numerous drug interactions and side effects, mainly visual changes (8-69\%, such as blurred vision, altered visual or color perception, and photophobia) and skin reactions (1-19\%) [79-81]. In addition, liver enzymes rise was observed in 4.3 to $26.5 \%$ of patients, but returned to normal at termination [79, 80]. In case of hepatic toxicity, it is important to exclude if the patient is part of the "poor 
metabolizer"population (genetically the activity of the CYP2C19 is low in 20\% of non-Indian Asians, consequently VRC blood levels may be up to 4 times higher than in "normal metabolizer" population). Due to accumulation of cyclodextrin (the IV VRC vehicle) when creatinine clearance is less than $50 \mathrm{ml} / \mathrm{min}$, oral route is recommended, if possible, as no adjustment in the dosage is necessary [80]. For patients with moderate liver disease, the loading dose will be the usual but the following doses will be halved [80]. No safety data is available in case of severe hepatic impairment.

Other triazoles (Posaconazole, Isavuconazole) are now available but their use against IPA is less documented and even more in COPD population. However, they exposed to fewer side effects so may replace VRC notably when liver enzymes increase [82-84].

Lipid formulations of amphotericin B (liposomal amphotericin B [AmBisome®], amphotericin B lipid complex [Abelcet ${ }^{\circledR}$ ], and amphotericin B colloidal dispersion [Amphotec $\left.{ }^{\circledR}\right]$ ) have been developed to lessen AmB-d related renal insufficiency. In addition, fewer hypokalemia, fever and infusion-related chills are encountered except with Amphotec ${ }^{\circledR}$ [85]. Although they are often suggested when VRC is contra-indicated, in COPD population there is no evidence of their effectiveness and their cost remains expensive.

Echinocandin (Caspofungin, Micafungin and Anidulafugin), another class of antifungals, shows some efficacy to control IPA [86, 87]. The drugs reported side-effects are scarce and the tolerance exhibits a favorable profile [87]. Another advantage of this antifungal class is that no dosage adjustment is needed for age $>18$ years, race, gender, renal impairment or haemodialysis, although the dosage of $35 \mathrm{mg}$ /day after a full loading dose is proposed when moderate hepatic insufficiency is present.

Despite an outcome improvement for patients under VRC [78], IPA prognosis remains limited. Therefore, combination therapy was suggested either with VRC-echinocandinor lipid formulation of AmB-echinocandin [88]. The former was most studied, however the available 
data remains rare and not convincing enough to recommend combination therapy at first-line treatment but may be as salvage regimen. The association between azoles and AmB is not indicated in view of in vitro studies showing some antagonism [89].

While some studies in hematological patients reported some efficacy of lung lesion resection $[5,90]$, the transposition of these results in COPD patients is often precluded due to the poor respiratory status. Hypothetically, wedge resection, by preventing the functional defect, could be discussed in selected cases, but no recent study sustenances this approach.

The place immunomodulation (such as growth factors, interferon- $\gamma$, leucocytes transfusion or vaccination) is anecdotic in COPD individuals as immunodepression is not the primum movens. 


\section{PROPHYLACTIC TREATMENTS AND PREVENTION}

In COPD population, considering that IPA diagnosis continue to be difficult and its calamitous prognosis, prophylactic treatment could be evoked but there are no consistent data to support its use despite some inputs from hematological patients [91, 92].

For the same reasons, prevention measures may be of importance. Despite insufficient data to firmly recommend a strategy, it seems reasonable to advice the following:

1. When the patient is hospitalized, to avoid exposure to Aspergillus spp. by wearing a highefficiency mask in case of Aspergillus exposure, by cleaning the surfaces to avoid dust accumulation, by erecting sealed barriers during construction works [93].

2. At home, the only proposition we can provide is to avoid exposure to knowned Aspergillus reservoirs (for instance: constructions, cellars or lofts, composts, ...). 


\section{PROGNOSIS}

Collecting the data available for COPD population until 2007, the mortality rate was $95 \%$ [1]. At that time, ICU admission did not seem to modify this poor prognosis $[15,44,94]$. In contrast, in more recent databases, a mortality rate between 55 to $60 \%$ is reported $[4,40]$. The explanation of this discrepancy could be that physicians now identify COPD by itself as a risk factor to contract IPA. As a result, suggestive symptoms are identified more rapidly and thus appropriate antifungals administered earlier. Second, the availibility of VRC, a more efficacious compound, could have modified the outcome (no VRC administered in the first reports). 


\section{CONCLUSIONS}

Although COPD patients may be colonized by Aspergillus, its presence in airways specimens could be the first sign of IPA motivating the performance of diagnostic procedures to offer the maximum chance of successful therapy.The first clinical symptoms are often persistant bronchospam and/or respiratory infection resistant to antibiotics. Thoracic ct-scan is recommended as chest X-ray is less sensitive to demonstrate mild pulmonary modifications, especially at the beginning. Bronchoscopy is usefull to ascertain IPA diagnosis as mucosal lesions could be observed allowing biopsies or to obtain galactomannan dosage in the BAL. The place of serological tests in the serum needs further studies and could not be routinely recommended. The use of criteria from the Clinical algorithm has demonstrated its usefulness in diagnosing IPA. Particularly when the patient is critically ill, and to provide the maximum chance of cure, appropriate treatment should be initiated immediately as well as diagnostic procedures started if IPA is suspected. Nowadays, VRC is the first-line antifungal and lipid formulations of AmB are the second choice. It could be an added value if COPD patients treated chronically by corticosteroids were given advice to avoid environmental exposure to Aspergillus spp. 


\section{REFERENCES}

1. Bulpa P, Dive A, Sibille Y. Invasive pulmonary aspergillosis in patients with chronic obstructive pulmonary disease. Eur Respir J 2007; 30: 782-800.

2. Patterson TF, Thompson GR 3rd, Denning DW, et al. Practice Guidelines for the Diagnosis and Management of Aspergillosis: 2016 Update by the Infectious Diseases Society of America. Clin Infect Dis 2016; 63: e1-e60.

3. Upton A, Kirby KA, Carpenter P, Boeckh M, Marr KA. Invasive aspergillosis following hematopoietic cell transplantation: outcomes and prognostic factors associated with mortality. Clin Infect Dis 2007;44:531-540.

4. Blot SI, Taccone FS, Van den Abeele AM, et al. A clinical algorithm to diagnose invasive pulmonary aspergillosis in critically ill patients. Am J Respir Crit Care Med 2012; 186: 56-64.

5. Caillot D, Casasnovas O, Bernard A, et al. Improved management of invasive pulmonary aspergillosis in neutropenic patients using early thoracic computed tomographic scan and surgery. J Clin Oncol 1997; 15: 139-147.

6. De Pauw B, Walsh TJ, Donnelly JP, et al. Revised definitions of invasive fungal disease from the European Organization for Research and Treatment of Cancer/Invasive Fungal Infections Cooperative Group and the National Institute of Allergy and Infectious Diseases Mycoses Study Group (EORTC/MSG) Consensus Group. Clin Infect Dis 2008; 46: 18131821.

7. Vandewoude K, Blot S, Benoit D, Depuydt P, Vogelaers D, Colardyn F. Invasive aspergillosis in critically ill patients: analysis of risk factors for acquisition and mortality. Acta Clin Belg 2004; 59: 251-257.

8. Vandewoude KH, Blot SI, Depuydt P, et al. Clinical relevance of Aspergillus isolation from respiratory tract samples in critically ill patients. Crit Care 2006; 10: R31.

9. Bulpa P, Bihin B, Dimopoulos G, et al. Which algorithm diagnoses invasive 
pulmonary aspergillosis best in ICU patients with COPD? Eur Respir J 2017; 50: 1700532 [https://doi.org/10.1183/13993003.00532-2017].

10. Taccone FS, Van den Abeele AM, Bulpa P, et al. Epidemiology of invasive aspergillosis in critically ill patients: clinical presentation, underlying conditions, and outcomes. Crit Care. 2015; 19: 7. doi: 10.1186/s13054-014-0722-7.

11. Tejerina EE, Abril E, Padilla R, et al. Invasive aspergillosis in critically ill patients: An autopsy study. Mycoses 2019; 62: 673-679.

12. Guinea J, Torres-Narbona M, Gijón P, et al. Pulmonary aspergillosis in patients with chronic obstructive pulmonary disease: incidence, risk factors, and outcome. Clin Microbiol Infect 2010; 16: 870-877.

13. Stuck AE, Minder CE, Frey FJ. Risk of infectious complications in patients taking glucocorticosteroids. Rev Infect Dis 1989; 11: 954-963.

14. Ader F, Nseir S, Le Berre R, et al. Invasive pulmonary aspergillosis in chronic obstructive pulmonary disease: an emerging fungal pathogen. Clin Microbiol Infect 2005; 11: 427-429. 15. Bulpa PA, Dive AM, Garrino MG, et al. Chronic obstructive pulmonary disease patients with invasive pulmonary aspergillosis: benefits of intensive care? Intensive Care Med 2001; 27: 59-67.

16. Rello J, Esandi ME, Mariscal D, Gallego M, Domingo C, Valles J. Invasive pulmonary aspergillosis in patients with chronic obstructive pulmonary disease: report of eight cases and review. Clin Infect Dis 1998; 26: 1473-1475.

17. Muquim A, Dial S, Menzies D. Invasive aspergillosis in patients with chronic obstructive pulmonary diseases. Can Respir J 2005; 12: 199-204.

18. Leav BA, Fanburg B, Hadley S. Invasive pulmonary aspergillosis associated with highdose inhaled Fluticasone. N Engl J Med 2000; 343: 586. 
19. Chow L, Brown NE, Kunimoto D. An unusual case of pulmonary invasive aspergillosis and aspergilloma cured with Voriconazole in a patient with cystic fibrosis. Clin Infect Dis 2002; 35: e106-e110.

20. Barouky R, Badet M, Saint Denis M, Soubirou JL, Philit F, Guerin C. Inhaled corticosteroids in chronic obstructive pulmonary disease and disseminated aspergillosis. Eur J Intern Med 2003; 14: 380-382.

21. Ali ZA, Ali AA, Tempest ME, Wiselka MJ. Invasive pulmonary aspergillosis complicating chronic obstructive pulmonary disease in an immunocompetent patient. $\mathrm{J}$ Postgrad Med 2003; 49: 78-80.

22. Bulpa P, Dive A, Delos M, Pouthier F, Delaunois L. Aspergillose pulmonaire invasive et infection à virus influenza chez des patients bronchitiques chroniques ? Rev Pneumol Clin 1995; 51: 300-301.

23. Shah MM, Hsiao EI, Kirsch CM, Gohil A, Narasimhan S, Stevens DA. Invasive pulmonary aspergillosis and influenza co-infection in immunocompetent hosts: case reports and review of the literature. Diagn Microbiol Infect Dis 2018; 91: 147-152.

24. Warnock DW, Hajjeh RA, Lasker BA. Epidemiology and prevention of invasive aspergillosis. Current Infectious Diseases Reports 2001; 3: 507-516.

25. Latgé JP. Aspergillus fumigatus and aspergillosis. Clin Microbiol Rev 1999; 12: 310350.

26. Schaffner A, Douglas H, Braude A. Selective protection against Conidia by mononuclear and against Mycelia by polymorphonuclear phagocytes in resistance to Aspergillus. J Clin Invest 1982; 69: 617-631.

27. Levitz SM. Overview of host defences in fungal infections. Clin Infect Dis 1992; 14: S37-S42. 
28. Roilides E, Uhlig K, Venzon D, Pizzo PA, Walsh TJ. Prevention of corticosteroidinduced suppression of human polymorphonuclear leukocyte-induced damage of Aspergillus fumigatus hyphae by granulocyte colony-stimulating factor and gamma Interferon. Infect Immun 1993; 61: 4870-4877.

29. Balloy V, Huerre M, Latgé JP, Chignard M. Differences in patterns of infection and inflammation for corticosteroid treatment and chemotherapy in experimental invasive pulmonary aspergillosis. Infect Immun 2005; 73: 494-503.

30. McCormick A, Loeffler J, Ebel F. Aspergillus fumigatus: contours of an opportunistic human pathogen. Cell Microbiol 2010; 12: 1535-43.

31. Szalewski DA, Hinrichs VS, Zinniel DK, Barletta RG. The pathogenicity of Aspergillus fumigatus, drug resistance, and nanoparticle delivery. Can J Microbiol 2018; 64: 439-453.

32. Latgé JP. The pathobiology of Aspergillus fumigatus. Trends in Microbiology 2001; 9: 382-389.

33. Philippe B, Ibrahim-Granet O, Prévost MC, et al. Killing of Aspergillus fumigatus by alveolar macrophages is mediated by reactive oxidant intermediates. Infect Immun 2003; 71: 3034-3042.

34. Ng TTC, Robson GD, Denning DW. Hydrocortisone-enhanced growth of Aspergillus spp.: implications for pathogenesis. Microbiology 1994; 140: 2475-2479.

35. Schaffner A. Therapeutic concentrations of glucocorticoids suppress the antimicrobial activity of human macrophages without impairing their responsiveness to gamma Interferon. J Clin Invest 1985; 76: 1755-1764.

36. Diamond RD. Inhibition of monocyte-mediated damage to fungal hyphae by steroid hormones. J Infect Dis 1983; 147: 160.

37. Lionakis MS, Kontoyiannis DP. Glucocorticoids and invasive fungal infections. Lancet $2003 ; 362: 1828-1838$. 
38. Berenguer J, Allende MC, Lee JW, et al. Pathogenesis of pulmonary aspergillosis.

Granulocytopenia versus Cyclosporine and Methylprednisolone-induced immunosuppression. Am J Respir Crit Care Med 1995; 152: 1079-1086.

39. Hartemink KJ, Paul MA, Spijkstra JJ, Girbes AR, Polderman KH. Immunoparalysis as a cause for invasive aspergillosis? Intensive Care Med 2003; 29: 2068-2071.

40. Barberan J, Sanz F, Hernandez JL, et al. Clinical features of invasive pulmonary aspergillosis vs. colonization in COPD patients distributed by gold stage. J Infect 2012; 65: 447-452.

41. Caillot D, Mannone L, Cuisenier B, Couaillier JF. Role of early diagnosis and aggressive surgery in the management of invasive pulmonary aspergillosis in neutropenic patients. Clin Microbiol Infect 2001; 7 (S2): 54-61.

42. Koulenti D, Garnacho-Montero J, Blot S. Approach to invasive pulmonary aspergillosis in critically ill patients. Curr Opin Infect Dis 2014; 27:174-183.

43. Koulenti D, Vogelaers D, Blot S. What's new in invasive pulmonary aspergillosis in the critically ill? Intensive Care Med 2014; 40: 723-726.

44. Meersseman W, Vandecasteele SJ, Wilmer A, Verbeken E, Peetermans WE, Van Wijngaerden E. Invasive aspergillosis in critically ill patients without malignancy. Am J Respir Crit Care Med 2004; 170: 621-625.

45. Pepys J, Riddell RW, Citron KM, Clayton YM, Short EI. Clinical and immunologic significance of Aspergillus fumigatus in the sputum. Am Rev Respir Dis 1959; 80: 167-180. 46. Yu VL, Muder RR, Poorsattar A. Significance of isolation of Aspergillus from the respiratory tract in diagnosis of invasive pulmonary aspergillosis. Results from a three-year prospective study. Am J Med 1986; 81: 249-254.

47. Comstock GW, Palmer CE, Stone RW, Goodman NL. Fungi in the sputum of normal men. Mycopathologia et Mycologia applicata 1974; 54: 55-62. 
48. Strimlan CV, Dines DE, Rodgers-Sullivan RF, Roberts GD, Sheehan WC. Respiratory tract Aspergillus. Clinical significance. Minnesota Medicine 1980; 63: 25-29.

49. Kahn FW, Jones JM, England DM. The role of bronchoalveolar lavage in the diagnosis of invasive pulmonary aspergillosis. Am J Clin Pathol 1986; 86: 518-523.

50. Andreas S, Heindl S, Wattky C, Möller K, Rüchel R. Diagnosis of pulmonary aspergillosis using optical brighteners. Eur Respir J 2000; 15: 407-411.

51. Nalesnik MA, Myerowitz RL, Jenkins R, Lenkey J, Herbert D. Significance of Aspergillus species isolated from respiratory secretions in the diagnosis of invasive pulmonary aspergillosis. J Clin Microbiol 1980; 11: 370-376.

52. Horvath JA, Dummer S. The use of respiratory-tract cultures in the diagnosis of invasive pulmonary aspergillosis. Am J Med 1996; 100: 171-178.

53. Greub G, Bille J. Aspergillus species isolated from clinical specimens: suggested clinical and microbiological criteria to determine significance. Clin Microbiol Infect 1998; 4: 710716.

54. Hope WW, Walsh TJ, Denning DW. The invasive and saprophytic syndromes due to Aspergillus spp. Med Mycol 2005; 43: S207-238.

55. Maertens J, Van Eldere J, Verhaegen J, Verbeken E, Verschakelen J, Boogaerts M. Use of circulating galactomannan screening for early diagnosis of invasive aspergillosis in allogeneic stem cell transplant recipients. J Infect Dis 2002; 186: 1297-1306.

56. Maertens J, Verhaegen J, Demuynck H, et al. Autopsy-controlled prospective evaluation of serial screening for circulating Galactomannan by a sandwich enzyme-linked immunosorbent assay for hematological patients at risk for invasive aspergillosis. J Clin Microbiol 1999; 37: 3223-3228. 
57. Ulusakarya A, Chachaty E, Vantelon JM, et al. Surveillance of Aspergillus galactomannan antigenemia for invasive aspergillosis by enzyme-linked immunosorbent assay in neutropenic patients treated for hematological malignancies. Hematology Journal 2000; 1: 111-116.

58. Williamson ECM, Oliver DA, Johnson EM, Foot ABM, Marks DI, Warnock DW. Aspergillus antigen testing in bone marrow transplant recipients. J Clin Pathol 2000; 53: 362366.

59. Weisser M, Rausch C, Droll A, et al. Galactomannan does not precede major signs on a pulmonary computerized tomographic scan suggestive of invasive aspergillosis in patients with hematological malignancies. Clin Infect Dis 2005; 41: 1143-1149.

60. Miceli MH, Maertens J. Role of Non-Culture-Based Tests, with an Emphasis on Galactomannan Testing for the Diagnosis of Invasive Aspergillosis. Semin Respir Crit Care Med 2015; 36: 650-661.

61. Leeflang MM, Debets-Ossenkopp YJ, Wang J, et al. Galactomannan detection for invasive aspergillosis in immunocompromised patients. Cochrane Database Syst Rev 2015: CD007394. doi: 10.1002/14651858.

62. He H, Ding L, Sun B, Li F, Zhan Q. Role of galactomannan determinations in bronchoalveolar lavage fluid samples from critically ill patients with chronic obstructive pulmonary disease for the diagnosis of invasive pulmonary aspergillosis: a prospective study. Crit Care 2012, 16: R138 doi: 10.1186/cc11443.

63. Fortún J, Martín-Dávila P, Gomez Garcia de la Pedrosa E, et al. Galactomannan in bronchoalveolar lavage fluid for diagnosis of invasive aspergillosis in non-hematological patients. J Infect 2016; 72: 738-744.

64. Zhang XB, Chen GP, Lin QC, Lin X, Zhang HY, Wang JH. Bronchoalveolar lavage fluid galactomannan detection for diagnosis of invasive pulmonary aspergillosis in chronic obstructive pulmonary disease. Med Mycol 2013; 5: 688-95. 
65. Boutboul F, Alberti C, Leblanc T, et al. Invasive aspergillosis in allogeneic stem cell transplant recipients: increasing antigenemia is associated with progressive disease. Clin Infect Dis 2002; 34: 939-943.

66. Caillot D, Couaillier JF, Bernard A, et al. Increasing volume and changing characteristics of invasive pulmonary aspergillosis on sequential thoracic computed tomography scans in patients with neutropenia. J Clin Oncol 2001; 19: 253-259.

67. Wheat LJ. Rapid diagnosis of invasive aspergillosis by antigen detection. Transpl Infect Dis 2003; 5: 158-166.

68. Mennink-Kersten MA, Ruegebrink D, Klont RR, et al. Bifidobacterial lipoglycan as a new cause for false-positive platelia Aspergillus enzyme-linked immunosorbent assay reactivity. $\mathrm{J}$ Clin Microbiol 2005; 43: 3925-3931.

69. Sulahian A, Touratier S, Ribaud P. False positive test for aspergillus antigenemia related to concomitant administration of piperacillin and tazobactam. N Engl J Med 2003; 349: 2366-2367.

70. Racil Z, Kocmanova I, Lengerova M, Winterova J, Mayer J. Intravenous PLASMALYTE as a major cause of false-positive results of platelia Aspergillus test for galactomannan detection in serum. J Clin Microbiol 2007; 45: 3141-3142.

71. Spriet I, Lagrou K, Maertens J, Willems L, Wilmer A, Wauters J. Plasmalyte: No Longer a Culprit in Causing False-Positive Galactomannan Test Results. J Clin Microbiol 2016; 54 : 795-797.

72. Cruciani M, Mengoli C, Loeffler J, et al. Polymerase chain reaction blood tests for the diagnosis of invasive aspergillosis in immunocompromised people. Cochrane Database Syst Rev 2015: CD009551. doi: 10.1002/14651858.

73. Pazos C, Pontón José, Del Palacio A. Contribution of (1®3)-b-D-glucan chromogenic assay to diagnosis and therapeutic monitoring of invasive aspergillosis in neutropenic adult 
patients: a comparison with serial screening for circulating galactomannan. J Clin Microbiol 2005; 43: 299-305.

74. Avni T, Levy I, Sprecher H, Yahav D, Leibovici L, Paul M. Diagnostic accuracy of PCR alone compared to galactomannan in bronchoalveolar lavage fluid for diagnosis of invasive pulmonary aspergillosis: a systematic review. J Clin Microbiol 2012; 50: 3652-3658.

75. Meersseman W, Lagrou K, Maertens J, et al. Galactomannan in bronchoalveolar lavage fluid: a tool for diagnosing aspergillosis in intensive care unit patients. Am J Respir Crit Care Med. 2008; 177: 27-34.

76. Zou M, Tang L, Zhao S, et al. Systematic review and meta-analysis of detecting galactomannan in bronchoalveolar lavage fluid for diagnosing invasive aspergillosis. PLoS One 2012; 7: e43347. doi: 10.1371/journal.pone.0043347.

77. Aquino VR, Nagel F, Andreolla HF, et al. The performance of real-time PCR, galactomannan, and fungal culture in the diagnosis of invasive aspergillosis in ventilated patients with chronic obstructive pulmonary disease (COPD). Mycopathologia 2012; 174: 163-169.

78. Herbrecht R, Denning DW, Patterson TF, et al. Voriconazole versus amphotericin B for primary therapy of invasive aspergillosis. N Engl J Med 2002; 347: 408-415.

79. Denning DW, Ribaud P, Milpied N, et al. Efficacy and safety of Voriconazole in the treatment of acute invasive aspergillosis. Clin Infect Dis 2002; 34: 563-571.

80. Hoffman HL, Rathbun RC. Review of the safety and efficacy of voriconazole. Expert Opin Investig Drugs 2002; 11: 409-429.

81. Levine MT, Chandrasekar PH. Adverse effects of voriconazole: Over a decade of use. Clin Transplant 2016; 30: 1377-1386. 
82. Walsh TJ, Raad I, Patterson TF, et al. Treatment of invasive aspergillosis with posaconazole in patients who are refractory to or intolerant of conventional therapy: an externally controlled trial. Clin Infect Dis 2007; 44: 2-12.

83. Maertens JA, Raad II, Marr KA, et al. Isavuconazole versus voriconazole for primary treatment of invasive mould disease caused by Aspergillus and other filamentous fungi (SECURE): a phase 3, randomised-controlled, non-inferiority trial. Lancet 2016; 387:760769.

84. Pettit NN, Carver PL. Isavuconazole: A New Option for the Management of Invasive Fungal Infections. Ann Pharmacother 2015; 49: 825-842.

85. Bowden R, Chandrasekar P, White MH, et al. A double-blind, randomized, controlled trial of amphotericin B colloidal dispersion versus amphotericin B for treatment of invasive aspergillosis in immunocompromised patients. Clin Infect Dis 2002; 35: 359-366.

86. Maertens J, Raad I, Petrikkos G, et al. Efficacy and safety of caspofungin for treatment of invasive aspergillosis in patients refractory to or intolerant of conventional antifungal therapy. Clin Infect Dis 2004; 39: 1563-1571.

87. Patil A, Majumdar S. Echinocandins in antifungal pharmacotherapy. J Pharm Pharmacol 2017; 69: 1635-1660.

88. Nivoix Y, Zamfir A, Lutun P, et al. Combination of caspofungin and an azole or an amphotericin B formulation in invasive fungal infections. J Infect 2006; 52: 67-74.

89. Meletiadis J, Petraitis V, Petraitiene R, et al. Triazole-polyene antagonism in experimental invasive pulmonary aspergillosis: in vitro and in vivo correlation. J Infect Dis 2006; 194 : 1008-1018.

90. Matt P, Bernet F, Habicht J, et al. Predicting outcome after lung resection for invasive pulmonary aspergillosis in patients with neutropenia. Chest 2004; 126: 1783-1788. 
91. Cornely OA, Maertens J, Winston DJ, et al. Posaconazole vs. fluconazole or itraconazole prophylaxis in patients with neutropenia. N Engl J Med 2007; 356: 348-359.

92. Wingard JR, Carter SL, Walsh TJ, et al. Randomized, double-blind trial of fluconazole versus voriconazole for prevention of invasive fungal infection after allogeneic hematopoietic cell transplantation. Blood 2010; 116: 5111-5118.

93. Patterson JE, Peters J, Calhoon JH, et al. Investigation and control of aspergillosis and other filamentous fungal infections in solid organ transplant recipients. Transpl Infect Dis 2000; 2: 22-28.

94. Garnacho-Montero J, Amaya-Villar R, Ortiz-Leyba C, et al. Isolation of Aspergillus spp. from the respiratory tract in critically ill patients: risk factors, clinical presentation and outcome. Crit Care 2005; 9: R191-199. 


\section{Table 1 DIAGNOSTIC CRITERIA FOR INVASIVE PULMONARY ASPERGILLOSIS ACCORDING TO THE COPD [1], CLINICAL [8] AND THE EUROPEAN ORGANIZATION FOR THE RESEARCH AND TREATMENT OF CANCER/MYCOSIS STUDY GROUP [6] ALGORITHMS.}

\section{COPD algorithm}

Proven invasive pulmonary aspergillosis

Histopathological or cytopathological examination, from needle aspiration or biopsy specimen obtained from any pulmonary lesion present for $<3$ months, showing hyphae consistent with Aspergillus and evidence of associated tissue damage, if accompanied by any one of the following:

1) Positive culture of Aspergillus spp. from any LRT sample.

2) Positive serum antibody/antigen test for $A$. fumigatus (including precipitins).

3) Confirmation that the hyphae observed are those of Aspergillus by a direct molecular, immunological method and/or culture.

Probable invasive pulmonary aspergillosis

As for proven IPA but without confirmation that Aspergillus is responsible (points 1,2 and 3 are not present or tested). OR

COPD patient, usually treated with steroids and severe according to GOLD (stage III or IV), with recent exacerbation of dyspnoea\#, suggestive chest imaging" (radiograph or CT scan; $<3$ months ${ }^{+}$) and one of the following:

1) Positive culture ${ }^{1}$ and/or microscopy for Aspergillus from LRT.

2) Positive serum antibody test for $A$. fumigatus (including precipitins).

3) Two consecutive positive serum galactomannan tests.

Possible invasive pulmonary aspergillosis

COPD patient, usually treated by steroids and severe according to GOLD (stage III or IV), with recent exacerbation of dyspnoea\#, suggestive chest imaging" (radiograph or CT scan; $<3$ months $^{+}$), but without positive Aspergillus culture or microscopy from LRT or serology.

Colonisation

COPD patient with positive Aspergillus culture from LRT without exacerbation of dyspnoea, bronchospasm or new pulmonary infiltrate.

\section{Clinical algorithm}

Proven invasive pulmonary aspergillosis

Microscopic analysis on sterile material: histopathologic, cytopathologic, or direct microscopic examination of a specimen obtained by needle aspiration or sterile biopsy in which hyphae are seen accompanied by evidence of associated tissue damage. Culture on sterile material: recovery of Aspergillus by culture of a specimen obtained by lung biopsy

Putative invasive pulmonary aspergillosis (all four criteria must be met)

1. Aspergillus-positive lower respiratory tract specimen culture (= entry criterion)

2. Compatible signs and symptoms (one of the following)

- Fever refractory to at least $3 \mathrm{~d}$ of appropriate antibiotic therapy

- Recrudescent fever after a period of defervescence of at least $48 \mathrm{~h}$ while still on antibiotics and without other apparent cause

- Pleuritic chest pain

- Pleuritic rub

- Dyspnea

- Hemoptysis

- Worsening respiratory insufficiency in spite of appropriate antibiotic therapy and ventilatory support

3. Abnormal medical imaging by portable chest X-ray or CT scan of the lungs

4. Either $4 \mathrm{a}$ or $4 \mathrm{~b}$

4a. Host risk factors (one of the following conditions)

- Neutropenia (absolute neutrophil count $<500 / \mathrm{mm} 3$ ) preceding or at the time of ICU admission

- Underlying hematological or oncological malignancy treated with cytotoxic agents

- Glucocorticoid treatment (prednisone equivalent, $>20 \mathrm{mg} / \mathrm{d}$ )

- Congenital or acquired immunodeficiency

4b. Semiquantitative Aspergillus-positive culture of BAL fluid ( + or ++ ), without bacterial growth together with a positive cytological smear showing branching hyphae

\section{Aspergillus respiratory tract colonization}

When $\geq 1$ criterion necessary for a diagnosis of putative IPA is not met, the case is classified as Aspergillus colonization. 


\section{EORTC/MSG algorithm}

Proven invasive pulmonary aspergillosis

Idem Clinical algorithm

Probable invasive pulmonary aspergillosis (all three criteria must be met)

1. Host factors (one of the following)

- Recent history of neutropenia $(<500$ neutrophils/mm3) for $>10 \mathrm{~d}$

- Receipt of an allogeneic stem cell transplant

- Prolonged use of corticosteroids at a mean minimum dose of $0.3 \mathrm{mg} / \mathrm{kg} / \mathrm{d}$ of prednisone equivalent for $>3 \mathrm{wk}$

- Treatment with other recognized T-cell immunosuppressants

- Inherited severe immunodeficiency

2. Clinical features (one of the following three signs on CT)

- Dense, well-circumscribed lesion(s) with or without a halo sign

- Air-crescent sign

- Cavity

3. Mycological criteria (one of the following)

- Direct test (cytology, direct microscopy, or culture) on sputum, BAL fluid, bronchial brush indicating presence of fungal elements or culture recovery Aspergillus spp.

- Indirect tests (detection of antigen or cell-wall constituents): galactomannan antigen detected in plasma, serum, or BAL fluid

Possible invasive pulmonary aspergillosis

Presence of host factors and clinical features (cf. probable invasive aspergillosis) but in the absence of or negative mycological findings.

Definition of abbreviations:

LRT: lower respiratory tract;

A. fumigatus: Aspergillus fumigatus;

COPD: Chronic Obstructive Pulmonary Disease

GOLD: Global Initiative for Chronic Obstructive Lung Disease;

CT: computed tomography.

\#: Exacerbation of dyspnoea and/or bronchospasm resistant to appropriate treatment including antibiotics;

": pulmonary lesion(s) unresponsive to appropriate antibiotics (refers to dose, route, spectrum and activity against cultured bacteria);

${ }^{+}$: pulmonary lesions, especially cavitary, present for $>3$ months are better classified as chronic pulmonary aspergillosis, unless direct tissue invasion is demonstrated;

${ }^{1}$ : standard or sabouraud culture, or molecular detection test when licensed.

$\mathrm{BAL}=$ bronchoalveolar lavage;

EORTC/MSG= European Organization for the Research and Treatment of Cancer/Mycosis Study Group;

$\mathrm{ICU}=$ intensive care unit. 
Number of patients: 56

$$
\begin{array}{lll}
\text { Sex: } & \text { - Male: } & 42(75 \%) \\
& \text { - Female: } & 14(25 \%) \\
\text { Age: } & \text { - Mean }( \pm \mathrm{SD}): & 65.5 \text { y.o. }( \pm 9.3) \\
& \text { - Median }(\mathrm{IQR}): & 66 \text { y.o. }[57-73]
\end{array}
$$

Steroids treatment: - At admission: 43

$\begin{array}{lr}\text { - systemic use: } & 40 \\ \text { - inhaled only: } & 3 \\ \text { - At hospital: } & 49 \\ \text { (systemic use) } & \\ \text { - None: } & 2 \\ \text { - NA: } & 5\end{array}$

Clinical signs (total pts available):

- Antibiotic resistant pneumonia (56): 53

- Dyspnoea exacerbation (48): 48

- Wheezing increase (28): $\quad 22$

- Fever $>38^{\circ} \mathrm{C}(39)$ : $\quad 15$

- Haemoptysis (12):

- Parietal pain: 4 reports

Tracheobronchitis during bronchoscopy (33) 6

Duration between symptoms and diagnosis: $\quad$ - Mean $( \pm \mathrm{SD}): \quad 12.5 \mathrm{~d} .( \pm 11.3)$ - Median [IQR]: $\quad 8.5$ d. [6-16.5]

Ventilation: - Invasive: 43

- Non invasive ventilation: 1

- None: $\quad 10$

- NA: 2

Total leucocytes: $\quad-<12$ 000: 10

- > 12 000: 30

- NA : $\quad 16$

Outcome (1): $\quad$ - Death: $\quad 53(=95 \%)$

- Survival: $\quad 3(=5 \%)$

Footnotes: $\quad \mathrm{SD}=$ standard deviation;

$\mathrm{IQR}=$ interquartile range;

$\mathrm{NA}=$ not available

(1) A total of 43 patients received treatment 

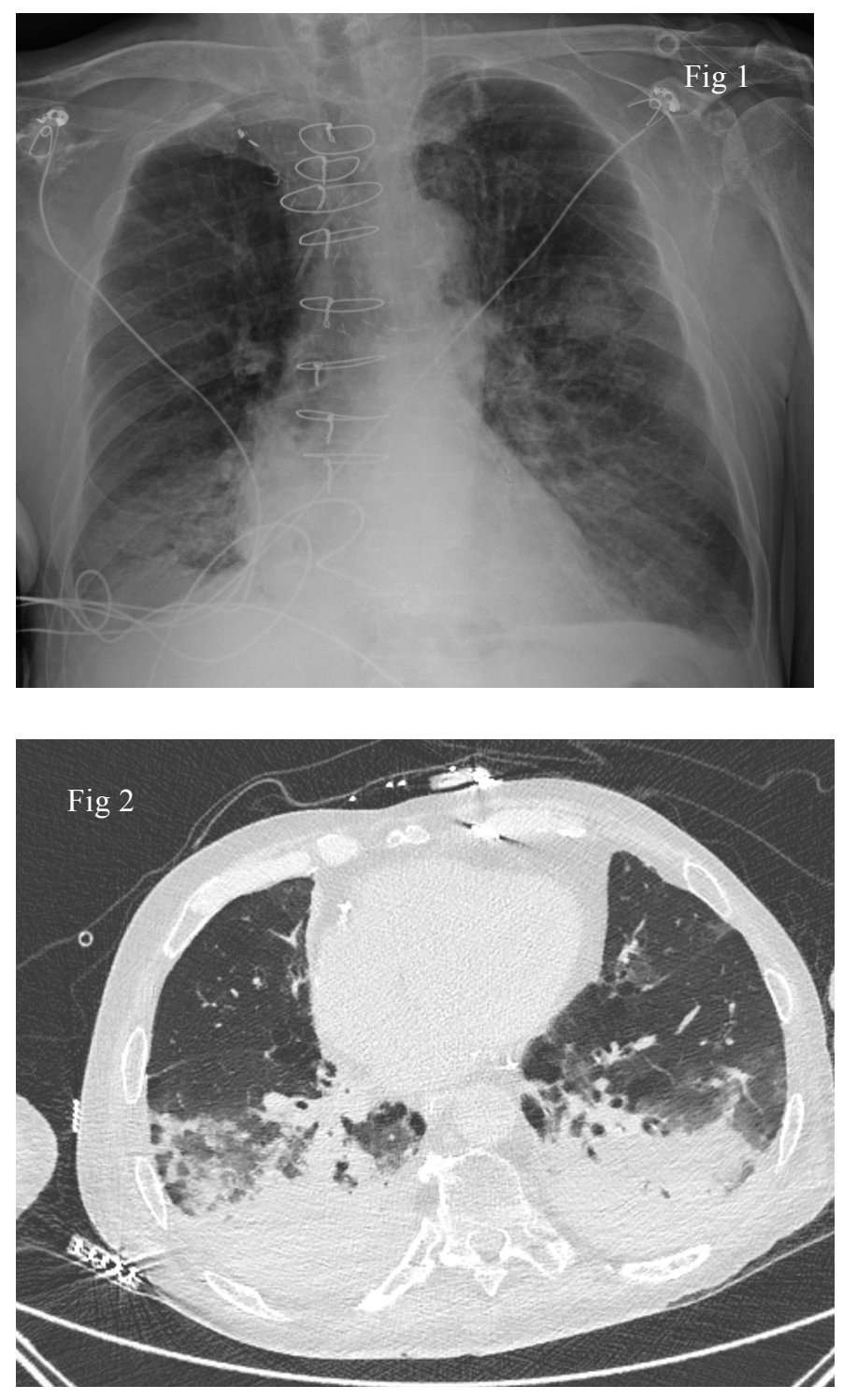

Fig 1. Chest X-ray showing bibasal non-specific consolidations.

Fig 2. Chest CT scan revealing bibasal non-specific consolidations. 

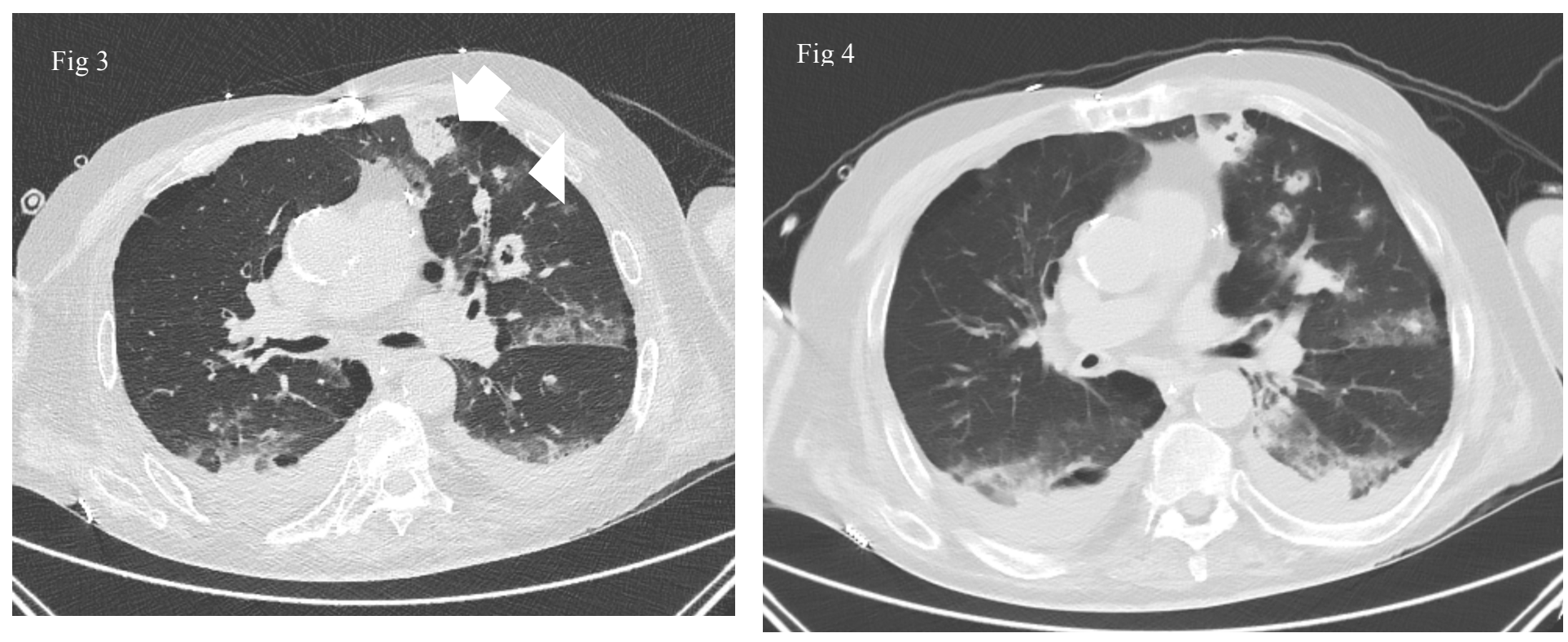

Fig 3. Chest CT scan showing wedge consolidation (arrow) of the left upper lobe corresponding to an infarcted zone, and one nodule with the "halo sign" (arrowhead).

Fig 4. The Chest CT scan showing multiple nodules. 


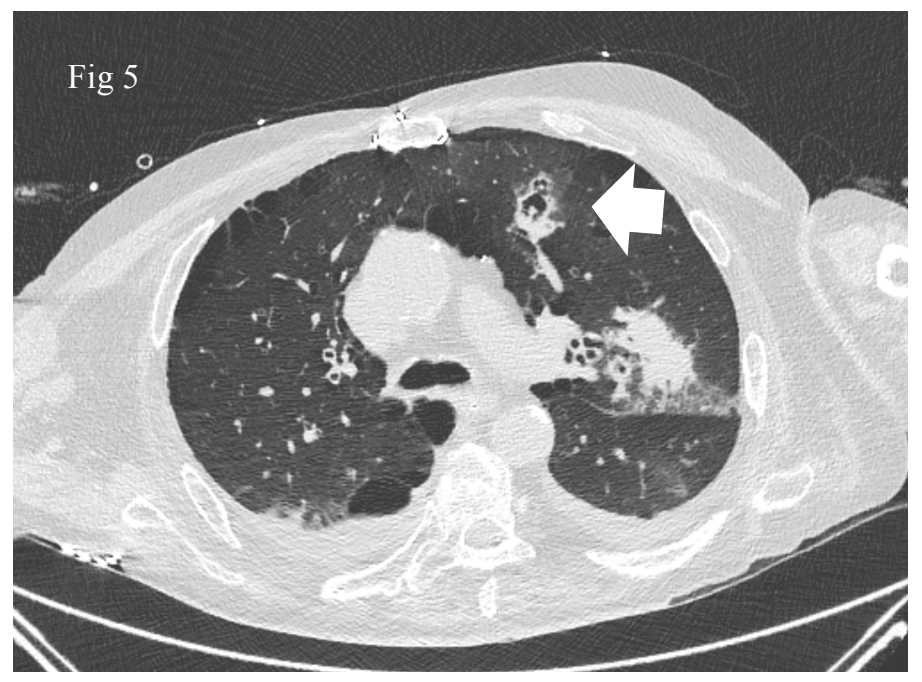

Fig 5. Chest CT scan showing a nodule with the "air-crescent sign": infarcted tissue surrounded by an air-filled space (arrow). 


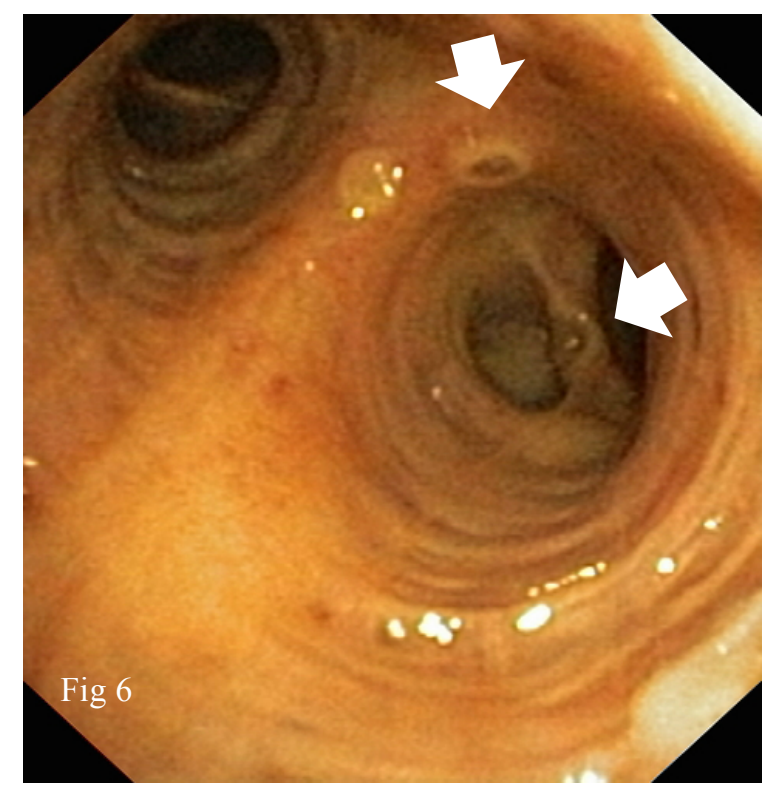

Fig 6. Endoscopic view of the left bronchus showing mucosa covered by few exulcerations, some with blackish center (arrow). 